\title{
Load Balancing Dynamic Source Routing Protocol Based on Multi-Path Routing
}

\author{
Zhongping Chen \\ Hunan Vocational College of Engineering, Changsha, China
}

\begin{abstract}
A HWMP improved routing protocol (HWMMRP) is proposed in this paper. The protocol adopts the integrated link state routing criterion algorithm LCCM providing small overhead, with factors such as bandwidth, queue length and noise interference fully considered. In order to solve the problem of the tree routing mechanism being easily congested at the root node, a multi-path multi-gateway shunting mechanism is applied. A multipath routing mechanism is also incorporated in the reactive routing mode. Both the new criteria and the protocol are simulated in the NS-2 environment, and are compared with comparable protocols. The experimental results show that our protocol can effectively avoid node congestion, and provides a better dynamic load balancing capability as well as a better performance than the standard HWMP and AODV protocols.
\end{abstract}

ACM CCS (2012) Classification: Networks $\rightarrow$ Network protocols $\rightarrow$ Network protocol design

Keywords: wireless mesh network, load balancing, link-state, multi-path routing, routing metric

\section{Introduction}

Wireless Mesh Network (WMN) is a self-organizing, self-healing and multi-hop wireless broadband network with many advantages such as wide coverage, high speed and low construction cost. With the continuous development of 802.11X series standards and wireless mobile communication technologies, WMN has been widely used [1-6]. However, WMN currently has problems such as security, capacity, network fairness, multi-hop routing, network load balancing, etc. Among them, optimal routing and network load balancing are the two main factors affecting WMN performance. Researchers have proposed a number of WMN routing protocols for the characteristics of WMN. These protocols have some improvement on the performance of WMN in different angles and environments, but there is still room for improvement worthy of further study.

Most of the early WMN routing protocols lack effective mechanisms for solving load balancing, which makes many network nodes load unevenly during operation, i.e. WMN resources cannot be fully utilized which results in poor performance of the entire network. In recent years, many researchers have proposed their own load balancing designs. The main research directions include multipath routing, load pre-judging, and multi-interface multi-channel communication. C. J. Lin et al. proposed a mesh network load balancing scheme based on resource allocation [7]. S. Waharte et al. proposed a load-aware Mesh network load balancing scheme [8]. Kharasani et al. proposed a load balancing routing algorithm for multi-radio wireless Mesh networks [9]. Feng et al. proposed a search for interference disjoint multipath routing protocol (MRAODV-DM) [10]. Liu et al. [11] proposed a load balancing routing protocol based on ant colony optimization algorithm in wireless mesh networks.

The hybrid wireless mesh routing protocol HWMP is a routing protocol in the IEEE 802.11s draft, it is a hybrid routing protocol. In the process of formulating the IEEE 802.11s standard, 
the working group proposed a hybrid wireless mesh routing protocol, a hybrid routing protocol. The main idea of the routing protocol is to combine on demand-driven and table-driven routing, with the flexibility of on demand-driven routing and the fast speed of table-driven routing, making it adaptable to more structures. The HWMP (Hybrid Wireless Mesh Protocol) protocol is a default routing protocol used in the IEEE 802.11s draft. It is a comprehensive routing protocol that combines a reactive routing protocol with a tree-based topology-based a priori routing protocol. It is also proposed for the basic characteristics of the mesh network, that is, most of the mesh nodes are relatively fixed, the mesh nodes of the backbone part are rarely changed, and some mesh nodes can be flexibly added and removed.

The Mesh Portal Point (MPP) can be used in the Mesh Point (MP) as the root node of the routing tree to quickly establish a route to the root node, and the MP itself can discover and maintain the optimal route. HWMP combines a priori routing and reversal routing. Tree-Based Routing (TBR) [12] and an improved protocol of AODV [13-20] (Radio-Metric AODV, RM-AODV) [21] are mainly used. HWMP has greatly improved the performance of WMN, but it relies too much on the processing of the root node, and it is easy to overload the root node. There are many places for improvement.

Based on the Hybrid Wireless Mesh Routing Protocol (HWMP), a new Low Comprehensive Cost Metric (LCCM) is proposed. Hybrid Wireless Mesh is used based on Multi-Path Routing Protocol (Hybrid Wireless Mesh based on Multi-Path Routing Protocol, HWMMRP) to improve network load balancing performance and achieve higher network throughput. Finally, on the NS-2 test platform, the performance of new criteria and protocols are tested and compared with other protocols.

\section{Methodology}

\subsection{Routing Metric}

The most important route metric (Metric) algorithm is to calculate the connection cost between two nodes at the minimum cost ac- cording to the actual situation of the network, and the best path is generated according to the calculation result. The hops implementation is very simple and requires no additional overhead. However, it does not consider the link quality and bandwidth, so the best path that is often obtained is not the best path in the true sense. The per-hop round tip time (RTT) measures the link quality by measuring the roundtrip time of the unicast detection packet. The expected number of transmissions ETX (Expected Transmission Count) is to estimate the number of retransmissions of the transmitted unicast data packets by counting the loss rate of the neighboring node broadcast test packets [22-24], its overhead is expensive. Expected Transmission Time (ETT) is an ETX that combines packet size and link bandwidth [25]. In the weighted cumulative ETT (WCETT), the increase in hop count is considered for resource consumption, taking into account overall and local optimization. The balance is also considered between throughput and latency. When new links are added to the route, the value of the medium time criterion will increase. WCETT can show better performance under multi-channel systems. The iAWARE routing metric is based on the signal-to-noise ratio of the wireless link [26], taking into account factors such as signal strength, background noise, and average yield factor. Additionally, it considers interference in the path and out-of-path interference. Similar to WCETT, iAWARE also considers the diversity of channel selection and performs well. However, the ETX mechanism always has a problem of frequent channel changes in the short term, and its formula calculation is not equally divided [26]. In summary, the current measurement mechanism has more or less different deficiencies, so it is necessary to find a routing metric mechanism that is small in cost, easy to calculate, and can accurately reflect the link quality. Obviously, there is still a lot of research work to be done.

A comprehensive criterion (Metric) is proposed in HWMMRP that is easy to calculate and has low overhead. Calculation of the criterion is based on the collection of link information on the node. It is not necessary to separately send the test data packet to collect information. The main collected information includes the link bandwidth, total amount of the data which is 
sent by the node, the queue length of the node data packet, and the noise node interference.

In the wireless network communication standard, the node can dynamically adjust the real-time bandwidth of the network card according to the current network link status, so the real-time bandwidth can reflect the communication link status of the node to a certain extent. The real-time bandwidth is obtained from the data link layer of the wireless routing node $i$, it is defined as $B W_{i}$. The number of packets that are successfully recived by node $i$ in the past period of time is recorded and it is defined as $R E_{i}$. The number of packets that are successfully sent by node $i$ is also recorded and it is defined as $S E_{i}$. The throughput capacity of the node unit time is defined as: $C O_{i}\left(C O_{i}=R E_{i}+S E_{i}\right)$.

The length of the node send and receive queues can also reflect the actual situation of link congestion. The length of the sending buffer queue of the node $i$ is $S Q_{i}$, the length of the limit sending buffer queue is $S Q_{\max }$, the length of the receiving buffer queue of the node $i$ is $R Q_{i}$, and the length of the limit receiving buffer queue is $R Q_{\max }$. The congestion index of a node is defined as $C_{i}$, and the calculation is shown in Equation 1:

$$
C_{i}=\frac{S Q_{i}}{S Q_{\max }} \times \alpha+\frac{R Q_{i}}{R Q_{\max }} \times \beta
$$

where $\alpha$ and $\beta$ are tunable values for the link quality degradation that is caused by moderately amplifying the congestion, and values multiplied by $\alpha$ and $\beta$ are related to the ratio of the queue length to the maximum length of the queue. The final value is determined as: when the ratio is $<0.5, \alpha$ and $\beta$ are set to values 1 ; when the 0.5 ratio is $<0.75, \alpha$ and $\beta$ are 5 ; when the ratio is $0.75, \alpha$ and $\beta$ are 10 . Here the values of $\alpha$ and $\beta$ are our empirical values. It is also possible to obtain the optimal values $\alpha$ and $\beta$ for the congestion degree.

Wireless signals are highly susceptible to interference, and it becomes an important factor that seriously affects the ability of the network to send and receive information. Since WMN is a multi-hop network, there are many signal collision domains. The transmission and reception of node signals are greatly affected by signals generated by external signals, especially by neighboring nodes. Therefore, the signal-to- noise ratio parameter of the node location can also reflect the current node ability to send and receive data. The node collects the noise signal strength and duration data in real time, and it starts to count the idle time $T_{i}$ when the noise intensity is lower than the normal transmission and reception signal threshold, and the statistics is terminated when the noise intensity becomes larger and exceeds the threshold.

According to the data that is collected above, the node $i$ integrated link state weight value can be defined as $W_{i}$, and its calculation method is shown in Equation 2:

$$
W_{i}=\left\lfloor\frac{C O_{i}}{B W_{i}} \times \mu\right\rfloor+C_{i}-T_{i} \times \delta
$$

where $\mu$ represents the influence coefficient of the node throughput capacity and the bandwidth ratio on the link state. The larger the coefficient, the greater the degree of influence, the value range is $1-10 ; \delta$ is the influence coefficient of the control channel idle time on the link state. When the time is in milliseconds and the statistical period is 1 second, the value ranges from 10 to 100 . In the case where the node status is good, the calculated value of $W_{i}$ may appear to be less than 1 or even a negative number. In order not to affect the calculation of other nodes in the link and to reflect the transmission and processing consumption of the node, when the calculation, less than 1 occurs, the situation is always 1 . The weight value of the node is calculated as follows:

$$
\left\{\begin{array}{l}
W_{i}<1: W_{i \text { new }}=1 \\
W_{i} \geq 1: W_{i \text { new }}=W_{i}
\end{array}\right.
$$

In summary, the calculation formula for the total weight $W_{n}$ of the path with $n$ nodes is the formula (3):

$$
W_{n}=\sum_{i=1}^{n}\left(\left\lfloor\frac{C O_{i}}{B W_{i}} \times \mu\right\rfloor+C_{i}-T_{i} \times \delta\right)
$$

\subsection{Routing Protocol Design}

The HWMP (Hybrid Wireless Mesh Protocol) protocol is a default routing protocol used in the IEEE $802.11 \mathrm{~s}$ draft. In a comprehensive rout- 
ing protocol, a reactive routing protocol is combined with a tree-based topology-based a priori routing protocol. Most of the mesh nodes are relatively fixed, the mesh nodes of the backbone part are rarely changed, and some mesh nodes can be flexibly added and removed.

When a mesh network is just being built, a Mesh node (generally a node connected to the wired domain) can be configured as a gateway node (Mesh Portal Point, MPP), which implements a tree-like routing network as a root node. Other mesh nodes (MPs) maintain the path to the root node a priori, while the root node maintains the path of each mesh node, whereby the mesh network establishes and maintains a priori bidirectional distance vector path tree. When the MP node has data to send, it will send it to the gateway node according to the path tree. If the data is sent to the external network, the MPP gateway will directly send the data packet through the external network link. The data is sent to other MP nodes in the mesh network, and the gateway forwards the data to the corresponding MP node. When the destination MP node receives the source MP node data from the intranet, it will send the source MP to the source MP. The node initiates the on-demand

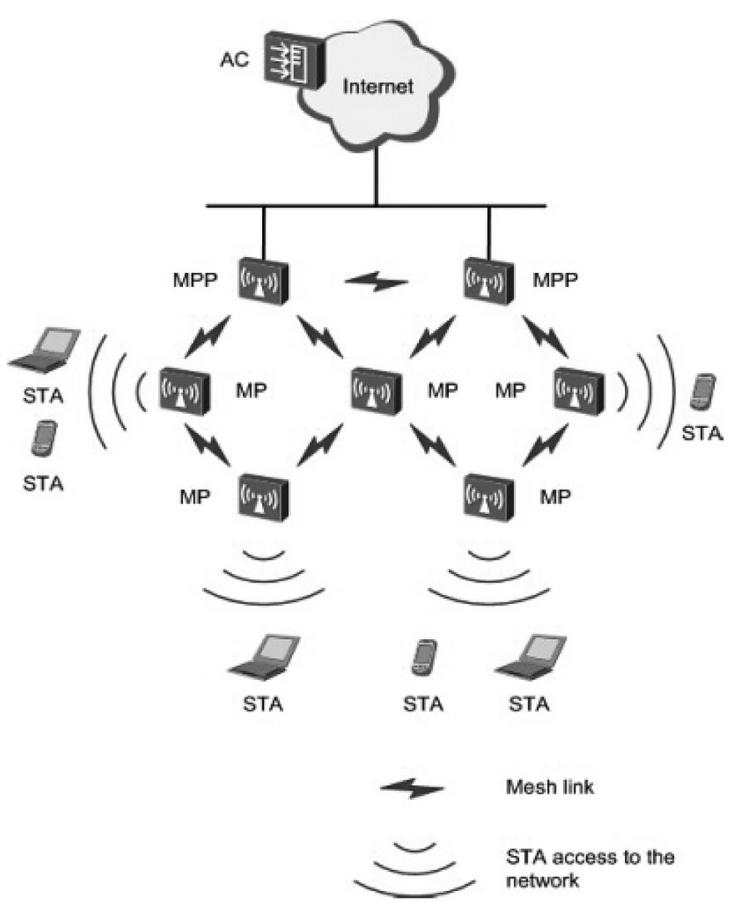

Figure 1. WMN network mesh link. path discovery mechanism and sends the corresponding route request packet. The source node adds the path of the destination MP node to the other MP node through the other MP nodes in the mesh network. If the new routing path is more efficient, then when the next data needs to be sent, it will be transmitted through this new internal path. Since the gateway node in a mesh network is far away from most MP nodes, the multi-hop transmission method directly connected through the nodes in the network generally saves network resources and is more efficient.

According to the characteristics of the multi-gateway hybrid WMN network environment, the routing of the extra-domain data stream service and the routing of the intra-domain data stream service are improved respectively based on the HWMP protocol, and a new route discovery and multi-path routing mechanism is proposed.

\subsubsection{Design of Extra-Domain Data Flow Routing Mechanism}

In the practical application of WMN, a lot of data needs to be sent to the external network, which must pass the MSH gateway node MPP, so a route is established in advance from the wireless mesh router node MP to the MPP.This way, the network working efficiency can be greatly improved. WMN network mesh link is shown in Figure 1. The tree-based routing mechanism TBR uses the MPP as the root node, and the gateway advertisement message RANN is periodically broadcast to maintain and update the routing tree. The RANN message contains the incremental sequence number and the parameter value of the MPP state to the gateway. After receiving the RANN message, the node first compares the sequence number and parameter values of the original record. When the sequence number in the RANN is greater than or equal to the original record value, and the parameter value is better than the original record, the node updates the record about the MPP. At the same time, the last hop in the RANN is used as its own parent node, the parameter value in the RANN is updated, and finally the message is continuously broadcast. When the reply identifier value is equal to 1 in the RANN, the receiving 


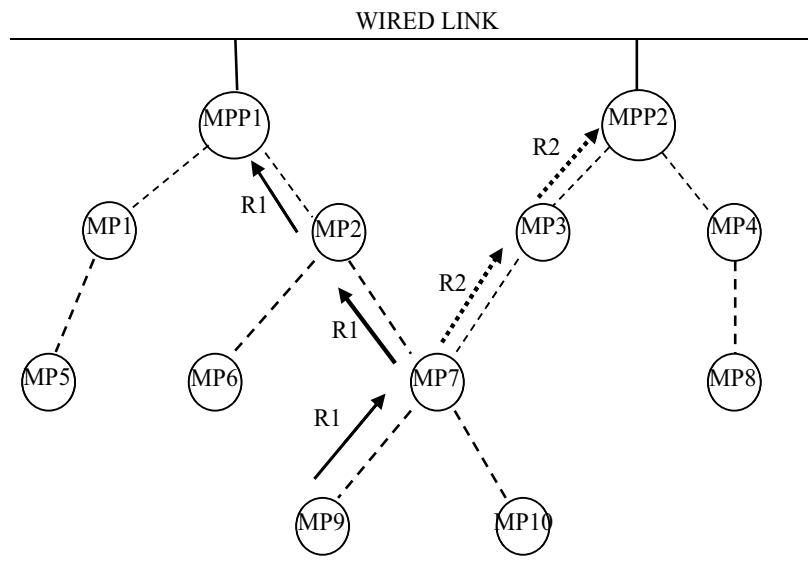

Figure 2. WMN network case, including two root nodes MPP1, MPP2 and 10 MPs.

node also needs to unicast a path reply message PREP to MPP, so that the MPP can learn the topology information of the entire network, and quickly provide any node to the MPP routing as needed. When there are multiple MPPs, the best MPP is selected according to the gateway node parameters in the RANN. The TBR routing mechanism enables each node in the network to quickly find an optimal path from itself to the root node (gateway) through the parent node. The root node can also record the path to the RREP message node through the RREP record. However, in the whole operation mechanism, the calculation and processing tasks of the root node are relatively heavy, and it is easy to become the bottleneck node of the whole network. The shunting of the root node load is the focus of this section.

A multipath and multiple gateway tree-based (MMGT) mechanism is proposed. The design idea is to transform the single MPP single-path data transmission mechanism into multiple MPPs in a multi-MPP mesh network environment. Multipath transmission effectively reduces the load of a single MPP, achieves load balancing, and improves network performance. As is shown in Figure 2, in MESH network en- vironment with dual gateways, node MP9 has extra-domain data transmission. If the TBR mechanism is used, MP9 data will only be transmitted to MPP1 along the path of R1, even if MPP1 is in a congested state and MPP2 is in an idle state. If the MMGT mechanism is used, two parent nodes can be established at the MP7 node, the two parent nodes belong to different root nodes, two paths are formed to the gateway node, thereby enabling offloading, reducing the burden on one MPP, and improving the performance of load balancing.

The difference between MMGT and TBR is as follows:

1. The establishment of a priori tree. The MMGT allows a node to have two or more parent nodes, and a forwarding node address entry (Diverger.Addr) is added in the RANN frame format, and is recorded as RANN-D. The frame format is shown in Figure 3. The nodes all add their own addresses in the RANN-D. The node receives the RANN-D from the same root node, in the same way as the TBR. However, if a node receives a RANN-D from a different root node, it first determines whether the Diverger.Addr of the message is the same as the recorded parent node. If they are the same, the serial number and the metric value are compared to see if it is necessary to replace the existing parent node record. If it is different, the root node and parent node information are recorded in the message as redundant routing information for future use.

2. Transmission of data frames. If the sending node has only one parent record, the sending method is the same as TBR. If there are multiple records of the parent node of the sending node, indicating that there are multiple MPPs and corresponding routes that can be used, the node sends

\begin{tabular}{|c|c|c|c|c|c|c|c|}
\hline Type & Length & Mark & TTL & Lifetime & Metric & Root. addr & Rootseq. Num \\
\hline 1 & 1 & 1 & 1 & 4 & 4 & 4 & 4 \\
\hline
\end{tabular}

(a) RANN

\begin{tabular}{|c|c|c|c|c|c|c|c|c|}
\hline Type & Length & Mark & TTL & Lifetime & Metric & Root. addr & Rootseq. Num & Diverger. Addr \\
\hline 1 & 1 & 1 & 1 & 4 & 4 & 4 & 4 & 4 \\
\hline
\end{tabular}

(b) RANN-D

Figure 3. Comparison of two gateway advertisement message frame formats. 
the data to multiple different MPPs and allocates the load of each path according to the metric value of each record.

\subsubsection{Reactive Routing Mechanism Design}

For the sending of data streams in the domain, HWMP will start the AODV routing mechanism. When node $X$ needs to send a message to node $Y$, it will first broadcast PREQ to find the path to $Y$. The intermediate node will relay the frame relay until $Y$ receives or has an intermediate node leading to the $Y$ node route, and then return PREP to node $X$. $X$ gets the path information to $Y$. However, the AODV protocol has relatively large defects in load balancing. The main reasons are:

1. The minimum number of hops is used as the criterion for the best path, it is difficult to effectively avoid congestion.

2. Single path routing is used. Many studies have shown that most load balancing mechanisms require the source node to have multiple paths to the destination node. In response to these shortcomings, an improved design is proposed in this sections, it is multipath AODV protocol (Multipath-Ad hoc On-demand Distance Vector Routing, MP-AODV).

In the MP-AODV, the proposed routing criteria algorithm in the previous section is used to replace the hop count as the best route metric. At the same time, MP-AODV modifies the route discovery mechanism of AODV, and the source node records the optimal multiple routing information that has been discovered, instead of storing a single route. When the destination node or the intermediate node of the known source node route sends the routing response packet, which is forwarded to the source node in unicast mode, the source node records the routing information in the routing table. If there are multiple paths to the destination node, the source node receives multiple different route response packets. In this case, the source node stores the path according to the metric value in the received route request message from low to high. In the routing table, only the three routing information with the best metric value are stored, and other discovered routes are discarded. When sending data, the sending task is as- signed according to the merit of the path in the routing table record. The specific transmission ratio is shown in Table 1.

Table 1. Data transmission allocation ratio of multipath routing.

\begin{tabular}{|c||c|c|c|}
\hline Path. Num & Path 1 & Path 2 & Path 3 \\
\hline \hline $\begin{array}{c}\text { Only one } \\
\text { path }\end{array}$ & $100 \%$ & $0 \%$ & $0 \%$ \\
\hline Two path & $\begin{array}{c}1-\text { Metric1/ } \\
\text { (Metric1 + } \\
\text { Metric2) }\end{array}$ & $\begin{array}{c}1-\text { Metric2/ } \\
\text { (Metric1 + } \\
\text { Metric2) }\end{array}$ & $0 \%$ \\
\hline Three path & $50 \%$ & $30 \%$ & $20 \%$ \\
\hline
\end{tabular}

Routing delays include domain name request delays, TCP connection setup delays, TCP connection release delays, and IP routing delays on individual gateways. If the user application uses the domain name of the other host instead of the IP address, the delay caused by the domain name resolution process of the peer IP address should be resolved before the application communicates. This is called the domain name request delay. The application hands the domain name to the local parser software, which first looks up the corresponding domain name in the local cache-address binding; if not found, the local parser constructs an inquiry message to the initial domain name server (local server). The domain name server answers a response message based on the analysis. The domain name server resolves the two-step method: When the initial server cannot find the domain name, it sends the query message to the root server for top-down search (the domain name server is organized into a tree hierarchy). The response delay of the local cache to the domain name request is determined by the CPU, memory, and external storage speed, which is relatively small. The response of the domain name server is related to the network load, the speed and load of the server. When it is necessary to start looking up a domain name from the root server, the transmission delay becomes a delay on the LAN, which is relatively large. The TCP connection setup delay refers to the time between when the transport service user requests to establish a connection and when a connection confirmation is received, which includes the 
processing delay of the remote transport layer. The connection release delay refers to the delay between when a transport layer user at one end initiates a release of a connection request and when the release of the other end actually occurs. Connection establishment and release delays are related to network load and server load. In the TCP/IP protocol, each IP packet is independently routed. IP path-finding delay refers to the path-finding delay of each IP packet from the source to the destination, including gateway path table processing delay and address resolution delay. Gateway address resolution is performed by the ARP (Address Resolution Protocol) provided by TCP/IP. Since the gateway routing is completed in the local machine and the address resolution is also performed in the local network, the IP path-finding delay is relatively small.

\section{Protocol Simulation and Discussion}

In order to verify the validity and correctness of the routing criteria for LCCM and HWMMRP protocols that are proposed in this paper, the protocol is implemented in NS-2, simulation experiments are carried out in three different business model environments, and the simulation results are analyzed. In order to form

Table 2. Simulation parameters.

\begin{tabular}{|c||c|}
\hline Simulation parameter & value \\
\hline \hline Network range & $800 \mathrm{~m} \times 800 \mathrm{~m}$ \\
\hline MAC layer & IEEE802.11b \\
\hline Transmission distance & $150 \mathrm{~m}$ \\
\hline RANN transmission interval & $3 \mathrm{~s}$ \\
\hline Data frame size & $1024 \mathrm{bit} / \mathrm{s}$ \\
\hline Bandwidth & $11 \mathrm{M} \mathrm{bit/s}$ \\
\hline Data frame generation rate & $30 \mathrm{frame} / \mathrm{s}$ \\
\hline Route calculation delay & $1 \mathrm{~ms}$ \\
\hline Coefficient $\mu$ & 10 \\
\hline Coefficient $\delta$ & 20 \\
\hline
\end{tabular}

an effective comparison, the HWMP protocol and the AODV protocol are also implemented. In the $800 \mathrm{~m} \times 800 \mathrm{~m}$ simulation scenario, the MPs are evenly arranged in a square topology, and two MPPs are arranged at the top of the two diagonals of the square. According to the characteristics of a typical mesh network, all MPs and MPPs set are fixed in the simulation. The simulation parameters are shown in Table 2. The parameters that are not marked are the default by the system.

The first simulation is set to the hybrid service mode, that is, the intra-domain data transmission service has the same probability as the extra-domain data transmission service. The intra-domain data service allows the mesh router to randomly select the destination address to send data packets, and the extra-domain service uses MPP as the destination node. The network scale has gradually increased from 10 router nodes to 60 , comparing the total throughput and average end-to-end delay of the HWMMRP, HWMP, and AODV protocols. The experimental results are shown in Figure 4. The network throughput refers to the total amount of data a node receives from the source node correctly in a unit of time [27]. The end-to-end delay is the total time required for the data packed to arrive to the destination node when sent from the sending node.

Figure 3(a) shows the changes in network throughput of the three protocols as the number of network nodes increases. It can be seen that HWMMRP has the strongest throughput, followed by HWMP and AODV. This shows that HWMMRP effectively utilizes multipath routing to improve network load balancing, thereby enhancing network throughput. Figure 3(b) shows the change in end-to-end delay for the three protocols. Among them, AODV has the longest delay time and HWMMRP is the shortest. As the number of nodes increases, the delay of AODV rises sharply, and the delay of HWMP rises more obviously. Only HWMMRP is relatively stable. The reason for this is because AODV is a purely reactive protocol, and each task needs to be routed through a broadcast, which causes a certain delay. HWMMRP and HWMP are hybrid protocols with a priori mechanism. Many extra-domain service data do not need to wait for route discovery, and can be sent directly, which reduces data transmission delay. There are more mul- 
tipath routing mechanisms in HWMMRP protocol than in HWMP protocol, so its performance is better when the load is aggravated.

After the proportion of the extra-domain data transmission service is adjusted to $80 \%$ or more and $20 \%$ or less of the total number of transmission tasks, the second and third simulation experiments were carried out respectively, and the experimental results are shown in Figure 5 and Figure 6.

Figure 5 shows that the performance indicators of HWMMRP and HWMP are much higher than AODV in the network environment that is dominated by extra-domain data services, but the gap between HWMMRP and HWMP is significantly smaller. The main reason is that both HWMMRP and HWMP have the same a priori mechanism. When the data is sent outside the domain, it can be sent directly, and the efficiency is high. Therefore, when most

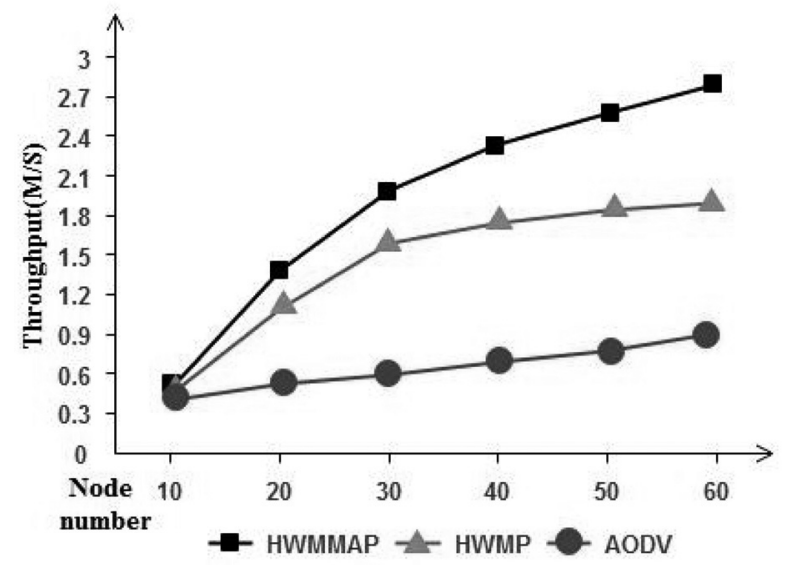

(a) Network throughput comparison

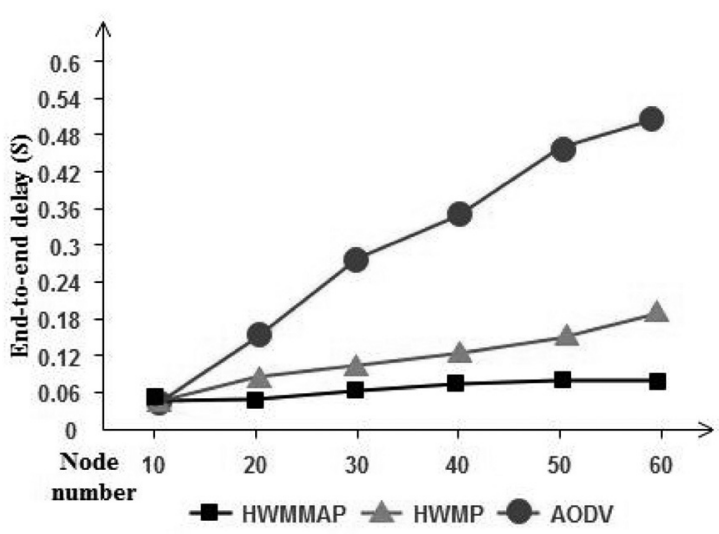

(b) End-to-end delay comparison graph

Figure 4. The first simulation experiment result. services are extra-domain services, the difference is small. The performance of HWMMRP is better than HWMP when the load is heavy. This is mainly because there are multiple MPPs in the simulation environment. In HWMMRP protocol, there is a data offloading mechanism, which makes its load balancing ability stronger than HWMP. Figure 6 shows that the gap between the three protocols becomes smaller in the network environment that is dominated by data services of the domain, especially the performance of HWMP and AODV is relatively close. As the main business is in the domain, the reactive mechanism is used for routing. The reaction mechanism of HWMMRP and HWMP is based on AODV. In particular, the reaction mechanism of HWMP is almost the same as that of AODV, so the performance is very close. However, Figure 6 also shows that the performance of HWMMRP still has obvious advantages, because HWMMRP uses a new criterion

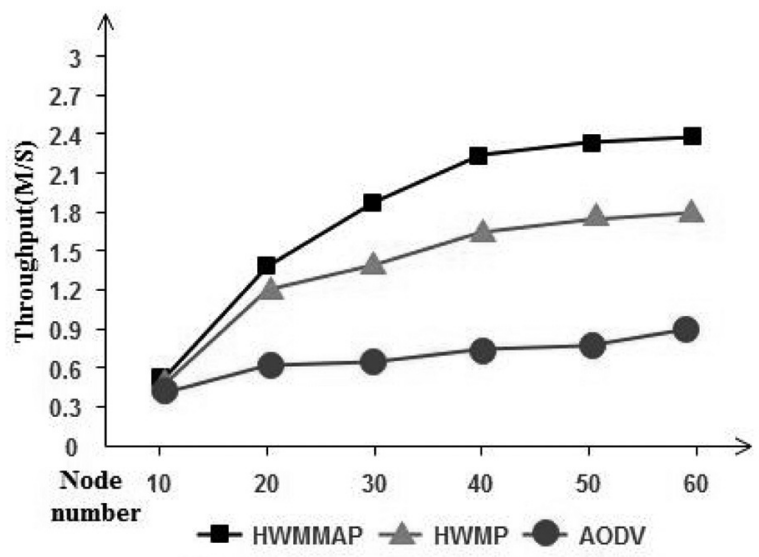

(a) Network throughput comparison

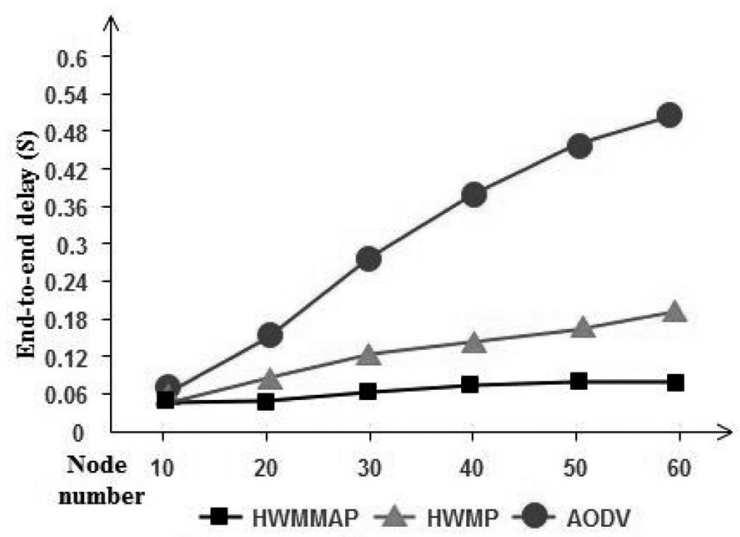

(b) End-to-end delay comparison graph

Figure 5. The second simulation experiment result. 
LCCM that can more accurately describe the link state of the node and has less overhead, and the mechanism of simultaneous multipath routing is used at the same time. Therefore, the load balancing capability of HWMMRP is also outstanding under the reactive mechanism and there is high network performance.

In the first simulation experiment environment, a root node and 7 intermediate nodes were selected, and the amount of data was counted during network operation that each node successfully received or forwarded packets on average. The result is shown in Figure 7. Among them, MPP1 is the root node, and MP1-MP7 are 7 mesh router nodes selected in order from the root node to the distance. It can be seen from Figure 7 that the throughput difference of each node is larger in AODV and HWMP environments, and the performance is more balanced under HWMMRP, which further illustrates the advantages of HWMMRP in load balancing capability.

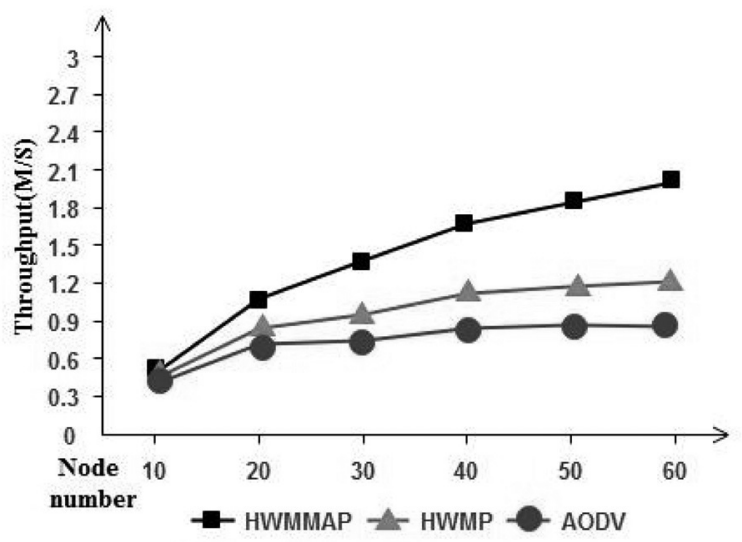

(a) Network throughput comparison

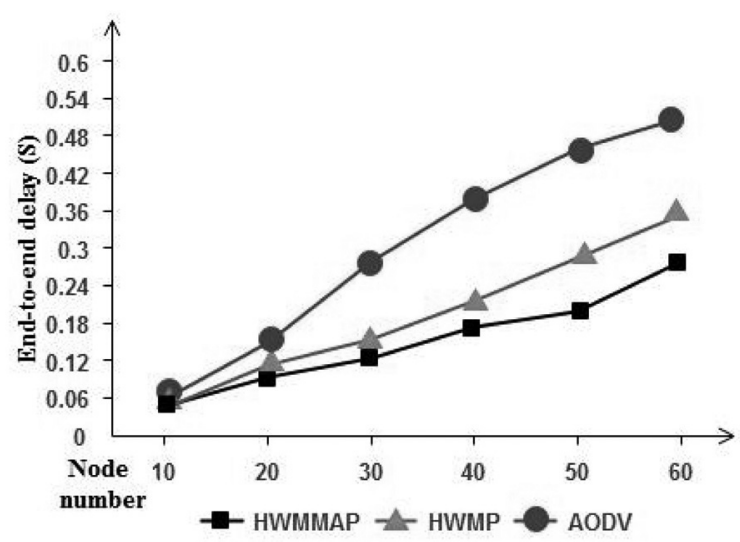

(b) End-to-end delay comparison graph

Figure 6. The third simulation experiment result.

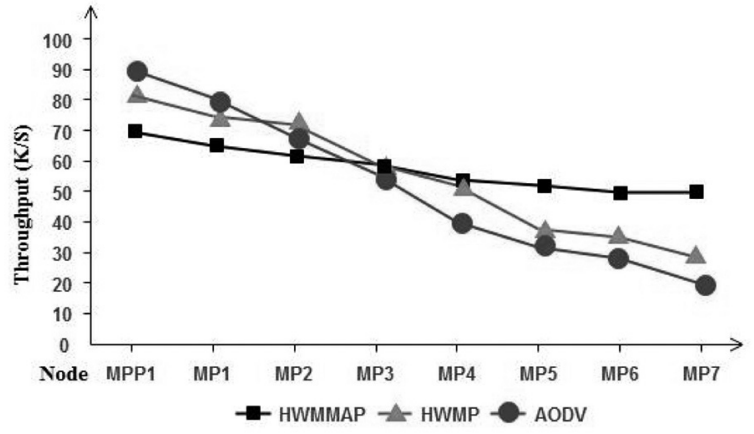

Figure 7. Throughput comparison on the nodes.

\section{Conclusion}

There are two basic working modes of multipath routing: (1) Multiple paths (simultaneous multipaths) are used at the same time; (2) The main path is used first; replacement paths (replace the multipath) are used after the main path have failed. To deal with the load balancing problem, the multipath mode is superior to the replacement multipath mode. In this respect, many existing multipath protocols are not desirable to some extent. For example, the disadvantage of MSR is that the processing overhead increases drastically when sending packets. The SMR has too many RREQ packets to transmit. In AOMDV, the data transmission often cannot use the shortest path. The load balancing mechanism of the shortest path routing algorithm has the problem of congesting network due to excessive load on some nodes. The widespread deployment and application requirements of the wireless network are rapidly increasing. There is a large space for the further optimization of multi-path routing protocols and further improvement of equilibrium mechanisms.

Aiming at the wireless mesh network, based on the research of the routing criterion with high overhead and high load balancing capability, a new routing criterion LCCM is proposed. Based on multipath routing, a hybrid wireless mesh routing protocol named HWMMRP is also proposed. Performance of the new criteria and protocol is tested and compared with other protocols on the NS-2 test platform. The simulation results show that LCCM criterion is used and multipath routing mechanism is integrated in the HWMMRP protocol. Compared with 
HWMP and AODV protocols, significant performance improvement is found, especially regarding load balancing capability. In the future, we will use this research as the cornerstone to further the optimization of the load balancing capability of wireless mesh networks and to study the integration of multi-channel mode, so as to additionally enhance the overall performance of wireless mesh networks.

\section{Acknowledgement}

This paper is sponsored by the National Natural Science Foundation project (51375484) of China and the 2017 School of Information College-level boutique online open course (single-chip principle and application).

\section{References}

[1] I. Akyildiz et al., "Wireless Mesh Networks: A Survey", Computer Networks, vol. 47, no. 4, pp. 445-487, 2005.

https://doi.org/10.1016/j.comnet.2004.12.001

[2] Y. Lu and X. Ding, "An Improved Dynamic Load Balancing Routing Protocol Based on Mesh Network", Journal of Computing and Information Technology, vol. 26, no. 4, pp. 223-234, 2018. http://dx.doi.org/10.20532/cit.2018.1004311

[3] N. M. Kharasani and Z. A. Zukarnain, "Performance Evaluation of Routing with Load-Balancing in Multi-Radio Wireless Mesh Networks", International Journal of Digital Content Technology and Its Applications, vol. 5, no. 2, pp. 64-71, 2011.

[4] W. J. Feng et al., "Multipath and Load Balancing Routing Protocol in Multi-Radio Wireless Mesh Network", Journal of System Simulation, vol. 23, no. 11, pp. 2518-2522, 2011.

[5] Y. B. Liu et al., "Load Balancing Routing Protocol Based on Traffic Prediction for Wireless Mesh Networks", Computer Science, vol. 44, no. 1, pp. 78-84, 2017.

http://dx.doi.org/10.11896/j.issn.1002-137X.2017.01.021

[6] IEEE802.11s.IEEE amendment: Mesh networking, IEEE P 802. 11 s/D1. 06,2007, Piscataway.

[7] C. J. Lin and C. F. Chou, "Route-Aware Load-Balanced Resource Allocation for Wireless Mesh Networks", WCNC 2007 at Direction of IEEE Communication Society Subject Matter Experts for Publication, 2007. http://dx.doi.org/10.1109/WCNC.2007.572
[8] S. Waharte et al., "Interference-Aware Routing Metric for Improved Load Balancing in Wireless Mesh Network", ICC 2008 at Direction of IEEE Communication Society Subject Matter Experts for Publication, 2008. http://dx.doi.org/10.1109/ICC.2008.561

[9] N. M. Al-Kharasani and Z. A. Zukarnain, "Performance Evaluation of Routing with Load-Balancing in Multi-Radio Wireless Mesh Networks", International Journal of Digital Content Technology and Its Applications, vol. 5, no. 2, pp. 64-71, 2011.

[10] W. J. Feng, et al., "Multipath and Load Balancing Routing Protocol in Multi-Radio Wireless Mesh Network", Journal of system Simulation, vol. 23, no. 11, pp. 2518-2522, 2011.

[11] C. X. Liu et al., "A Load Balanced Routing Protocol Based on Ant Colony Algorithm for Wireless Mesh Network", in Proc. of the 5th International Conference on Genetic and Evolutionary Computing (ICGEC 2011), Kinmen, China, 2011, pp. 295-298.

[12] A. Raniwala and T. Chiueh, "Architecture and Algorithms for an IEEE 802.11-based Multi-Channel Wireless Mesh Network", in Proc. of Int. Conference on the IEEE Computer and Communications Societies (INFOCOM'05), 2005, pp. 2223-2234.

http://dx.doi.org/10.1109/INFCOM.2005.1498497

[13] C. Perkins et al., "Ad Hoc On-Demand Distance Vector (AODV) Routing", IETF Experimental RFC 3561, 2003.

www.ietf.org

[14] K. T. Chai et al., "Load Balanced Routing Protocols for Ad Hoc Mobile Wireless Networks", IEEE Communications Magazine: Articles, News, and Events of Interest to Communications Engineers, vol. 8, no. 8, pp. 78-84, 2009. http://dx.doi.org/10.1109/MCOM.2009.5181896

[15] J. Su Jing and W. Guo, "Summarization of Wireless Mobile Ad Hoc Network Routing Protocols", China Measurement Technology, vol. 35, no. 2, pp. 91-93, 2005.

http://dx.doi.org/10.3969/j.issn.1674-5124.2005.02.034

[16] C. Perkins et al., "Ad Hoc on Demand Distance Vector (AODV) Routing", in Proc. of The Internet Engineering Task Force (IETF), 2003. http://dx.doi.org/doi:10.17487/RFC3561

[17] D. Johnson et al., "The Dynamic Source Routing Protocol (DSR) for Mobile Ad Hoc Networks for IPv4", in Proc. of RFC 4728, 2007. http://tools.ietf.org/html/rfc4728

[18] S. J. Lee and M. Gerla, "Split Multipath Routing with Maximally Disjoint Paths in Ad Hoc Network", in Proc. of IEEE Int. Conference on Communications, 2001, pp. 3201-3205. 
[19] Y. T. Shu et al., "Multipath Source Routing in Wireless Ad Hoc Networks", Acta Electronica Sinica, vol. 30, no. 2, pp. 279-282, 2002.

http://dx.doi.org/10.3321/j.issn:0372-2112.2002.02.034

[20] S. Lei and G. Lindong, "A Node Disjoint Multipath Routing Protocol in Mobile Ad Hoc Networks", Computer Engineering and Applications, vol. 41, no. 3, pp. 159-161, 2005.

http://dx.doi.org/10.3321/j.issn:1002-8331.2005.03.051

[21] IEEE Task Group S, "Simple Efficient Extensible Mesh (SEE-Mesh) Proposal", IEEE P802.11 Wireless LANs, IEEE Document 802.1105/0562ro, 2005.6.

[22] S. J. D. Couto et al., "A High-Throughput Path Metric for Multi-Hop Wireless Routing", 9th Annual International Conference on Mobile Computing (MobiCom 03), 2003. http://dx.doi.org/10.1145/938985.939000

[23] S. Waharte et al., "Interference-Aware Routing Metric for Improved Load Balancing in Wireless Mesh Network" in Proc. of IEEE Int. Conference on Communications, 2008.

http://dx.doi.org/10.1109/ICC.2008.561

[24] L. MA and M. K. Denko, "A Routing Metric for Load Balancing in Wireless Mesh Networks", in Proc. of IEEE 21st Int. Conference on Advanced Information Networking and Applications Workshops (AINAW'07), 2007.

http://dx.doi.org/10.1109/AINAW.2007.50

[25] R. Draves et al., "Routing in Multi-Radio, MultiHop Wireless Mesh Networks", in MobiCom '04: Proceedings of the 10th Annual International Conference on Mobile Computing and Networking, Philadelphia, USA, ACM Press, 2004, pp. 114-128.

http://dx.doi.org/10.1145/1023720.1023732

[26] A. P. Subramanian et al., "Interference Aware Routing in Multi-Radio Wireless Mesh Networks", Second IEEE Workshop on Wireless Mesh Networks, 2006, pp. 25-28. http://dx.doi.org/10.1109/WIMESH.2006.288620

[27] D. Senthilkumar and A. Krishnan, "Throughput Analysis of IEEE802.11 Multirate WLANs with Collision Aware Rate Adaptation Algorithm", Journal of Automation and Computing, vol. 7, no. 4, pp. 571-577, 2010. http://dx.doi.org/10.1007/s11633-010-0542-4
Received: April 2019

Revised: July 2019

Accepted: July 2019

Contact address:

Zhongping Chen

Hunan Vocational College of Engineering

Changsha

Hunan Province

China

e-mail: 125186223@qq.com

ZHONGPING CHEN received the BSc degree in applied electronic technology from Hunan Normal University, China, in 2002, and the MSc degree in software engineering from Tongji University, China, in 2011. $\mathrm{He}$ is currently teaches electronic information engineering technology at Hunan Engineering Vocational and Technical College, China. His main research interests are automatic control, embedded and network optimization. 